\title{
Factors associated with waiting time on the liver transplant list: an analysis of the United Network for Organ Sharing (UNOS) database
}

\author{
Judy A. Trieu, Mohammad Bilal, Bashar Hmoud \\ University of Texas Medical Branch, Galveston, TX, USA
}

\section{Abstract}

\section{Introduction}

Liver transplantation is an important treatment for acute liver failure and end-stage liver disease or cirrhosis. The United Network for Organ Sharing (UNOS) is a non-profit organization that serves in allocating organs in the United States. Previously, prioritization of liver transplant recipients was based on time spent on the waiting list. However, since the transitioning to disease severity-based allocation of the organ, transplantation rates have improved, with a concomitant

Division of Gastroenterology and Hepatology, Department of Internal Medicine, University of Texas Medical Branch, Galveston, TX, USA

Conflict of Interest: None

Correspondence to: Mohammad Bilal, MD, 7400 Jones Drive, Apt 724, Galveston, TX 77551, USA, e-mail: billa17@hotmail.com

Received 8 September 2017; accepted 22 October 2017; published online 27 November 2017

DOI: https://doi.org/10.20524/aog.2017.0217 reduction of mortality on the waiting list $[1,2]$. Due to the limited supply of livers, there are still over 14,000 candidates waiting for transplantation and only just over $50 \%$ received an organ in 2016 [3]. The increasing demand on limited resources results in longer waiting times on the liver transplantation list.

The model for end-stage liver disease (MELD) score is a scoring system initially developed following transjugular intrahepatic porto-systemic shunt (TIPS) placement $[4,5]$. It was later adjusted and validated to predict 3-month mortality in all patients with cirrhosis with good accuracy [5]. Serum bilirubin, serum creatinine, and international normalized ratio (INR) constitute the MELD score and each component is independently weighed to reach the overall score. UNOS adopted the MELD score in 2002 as the main tool for prioritization of patients awaiting transplantation, based on disease severity. Although the MELD score improved predictions of mortality risk while on the waiting list, many independent factors expected to increase waiting time on the transplant list have not been fully evaluated.

Hyponatremia is a complication from liver cirrhosis, resulting from hepatorenal syndrome, ascites, and chronic 
diuretic use [6]. Although serum sodium (Na) has not been shown to be an independent predictor of death, it is associated with higher mortality [6,7]. The United Kingdom MELD (UKELD) incorporates sodium into its calculation and scoring for liver allocation. The modified MELD-Na takes serum sodium into account. Albumin is another factor included in the Child-Turcotte-Pugh score, which was a disease-severity classification used concomitantly with the aforementioned waitlist-time model. Hypoalbuminemia is a marker for malnutrition and, in liver cirrhosis, it has been shown to be associated with poor liver function; however, there are conflicting reports regarding the validation of albumin as an independent risk factor for mortality $[8,9]$.

There are other factors that appear to play a role in disease severity. For instance, having hepatocellular carcinoma can allocate MELD exception points and in turn decrease waiting times. Likewise, the need for intensive care and/or mechanical ventilation can also prioritize candidates on the liver transplant list. Factors not associated with disease severity, such as transplant region, ethnicity, candidate's $\mathrm{ABO}$ blood type, primary source of payment, and TIPS placement, may also contribute to liver allocation; however, the impact on waiting list time is not fully understood.

This study aimed to evaluate the factors affecting waiting times for liver transplantation in the United States using the UNOS database.

\section{Patients and methods}

\section{Study population}

We used the UNOS database for the years 2002-2015 for the present analysis. Patients listed for liver transplantation in the UNOS database within the age range of 12-75 years were included. Listings prior to the implementation of MELD scoring in 2002 were excluded.

\section{Selecting variables}

The variables included in the analysis are listed in Table 1. Among these were age at listing, body mass index (BMI), as well as laboratory data including serum bilirubin, albumin, sodium, creatinine, and INR. These laboratory values were included since they constitute the MELD and are also used to allocate patients on the transplant list. Serum sodium was also included since it is part of the MELD-Na scoring system. Serum albumin is used to assess the severity of liver disease in another model, Child-Turcotte-Pugh scoring, and hence it was added to our model. As for age, it is an independent risk for mortality and might influence the duration of being on the transplantation list. Finally, we included BMI, as both obesity and age are thought to increase the risk of progression of cirrhosis in liver disease from other etiologies [10].

Other variables signifying the complications due to liver disease, such as ascites, hepatocellular carcinoma (HCC), and the need for mechanical ventilation, were also incorporated in the analysis. Other factors included ABO blood type, transplant region, primary payer status, ethnicity, and listing for dualorgan transplantation. Transplantation across ABO blood groups has been used in emergency settings; however, given the difference in distribution amongst the $\mathrm{ABO}$ groups in the population, the availability of organs is expected to be different and can result in differences in waiting times. In addition, receiving MELD exception points and current mechanical ventilation are expected to expedite liver transplantation. The presence and degree of ascites is used to estimate how advanced is the liver disease and is expected to decrease the time on the liver transplant waiting list. On the other hand, patients listed for dual liver and kidney transplantation are expected to have a longer waiting time. The effects of transplant region, ethnicity, type of primary payer status and TIPS placement are also not fully understood.

\section{Outcome measures}

Our primary outcome measure was waiting time on the transplant list.

\section{Statistical analysis}

SAS version 9.4 was used for the analysis. The dependent variable was tested for the normality assumption and transformed as needed. As the number of days waiting on the transplant list was highly skewed, logit transformation was used; further testing utilizing univariate analysis showed the appropriateness of the normality assumption after the transformation. All the continuous variables were tested for collinearity and variance inflation factors were measured in the final model. To build the model, a backward elimination method was used. If the P-value was $<0.1$, the variable was kept in the model. Outliers were evaluated using scatter plots, studentized residuals, and the evaluation of influential outliers using Cook's distance. Variables were considered statistically significant in the analysis against waiting list time if the P-value was $<0.05$.

\section{Results}

A total of 75,771 candidates were identified and included in the study. Of these, 16,438 patients had missing values. Table 1 summarizes the patient characteristics and variables recorded. The mean age was $51.9 \pm 11.45$ years and $66.2 \%$ were male. As regards ethnicity, Caucasians received the highest number of liver transplants (72.1\%), followed by African American (9.5\%), Hispanic (12.9\%), and other races (5.4\%). Mean BMI was $28.67 \mathrm{~kg} / \mathrm{m}^{2}$. The most common blood type among candidates was "O-positive", representing $44 \%$ of the patient population. The highest number of patients were listed in region 3 (17.1\%). 
Table 1 Demographics, clinical, biochemical, financial, and geographical factors of candidates on the UNOS transplant list in 2016

\begin{tabular}{|c|c|c|c|}
\hline Transplant candidate factors & Value & Source of pay & $\mathrm{n}(\%)$ \\
\hline \multicolumn{4}{|l|}{ Age (years) $($ mean $\pm \mathrm{SD})$} \\
\hline Recipient sex n (\%) & $51.9 \pm 11.45$ & Private & $53,228(61.92 \%)$ \\
\hline Male & $56,940(66.24 \%)$ & Medicaid & $12,234(14.23 \%)$ \\
\hline Female & $29,018(33.76 \%)$ & MedicareFFS & $7,760(9.03 \%)$ \\
\hline Ethnicity n (\%) & & MedicareCh & $4,859(5.65 \%)$ \\
\hline Caucasian & $61,983(72.11 \%)$ & CHIP & $37(0.04 \%)$ \\
\hline African American & $8167(9.5 \%)$ & Department/VA & $1,677(1.95 \%)$ \\
\hline Hispanic & $11,162(12.99 \%)$ & Listing region n (\%) & \\
\hline Other & $4,646(5.4 \%)$ & 1 & $3,531(4.11 \%)$ \\
\hline BMI kg/m² (mean) & 28.67 & 2 & $10,082(11.73 \%)$ \\
\hline Blood group n (\%) & & 3 & $14,722(17.13 \%)$ \\
\hline A & $32,309(37.59 \%)$ & 4 & $8,020(9.33 \%)$ \\
\hline $\mathrm{AB}$ & $4,315(5.02 \%)$ & 5 & $12,006(13.97 \%)$ \\
\hline B & $11,503(13.38 \%)$ & 6 & $2,347(2.73 \%)$ \\
\hline $\mathrm{O}$ & $37,831(44.01 \%)$ & 7 & $7,875(9.16 \%)$ \\
\hline Biochemical (mean \pm SD) & & 8 & $5,897(6.86 \%)$ \\
\hline Bilirubin $(\mathrm{mg} / \mathrm{dL})$ & 6.66 & 9 & $5,277(6.14 \%)$ \\
\hline Creatinine $(\mathrm{mg} / \mathrm{dL})$ & 1.4 & 10 & $7,513(8.74 \%)$ \\
\hline INR & 1.74 & 11 & $8,688(10.11 \%)$ \\
\hline Albumin (g/dL) & 3 & TIPS n (\%) & 6007 (6.99\%) \\
\hline \multirow[t]{3}{*}{ Sodium (mEq/L) } & 136.09 & Dual transplants (n) & \\
\hline & & Liver/Kidney & 5,677 \\
\hline & & Liver/Intestine & 459 \\
\hline
\end{tabular}

BMI, body mass index; INR, international normalized ratio; CHIP, children's health insurance program; VA, veterans affairs; TIPS, transjugular intrahepatic portosystemic shunt

After transformation, the normality assumption was met for all variables. No influential outliers were identified and Cook's distance was less than 0.1 for all the values. The variables included in the final analysis of significance correlated against time on the waiting list are listed in Table 2.

Candidates identified as Caucasian or Hispanic had significantly longer waiting times. BMI was also significant in determining waiting time on the transplant list. The components of the MELD score, namely the levels of bilirubin, INR, and creatinine, were associated with a shorter waiting time on the transplant list. Hypoalbuminemia and hyponatremia did show significance in affecting the time on the waiting list, but not as much as the core MELD measures. The presence of complications of liver disease that contribute to disease severity, such as the presence of ascites, HCC, and the need for life support, predicted shorter waiting times. However, patients who underwent TIPS placement spent longer times awaiting liver transplantation.

Other factors associated with shorter waiting times were the need for mechanical ventilation and the region of transplantation, with region 3 being predictive of the shortest waiting time. $\mathrm{ABO}$ blood types also influenced waiting time, with the $\mathrm{O}$ subtype associated with the longest waiting time.
Primary payer status also influenced waiting time on the liver transplant list.

\section{Discussion}

The evolution of models for liver allocation, particularly with UNOS, has demonstrated the importance of data-driven validation of factors that will impact candidates' survival. The current model is severity-driven; thus, "sicker" patients should theoretically have shorter waiting times on the transplant list. As expected, the components of the MELD score, which are the core of the UNOS model for liver allocation, were highly predictive of a shorter waiting time. Although the adoption of MELD scoring by UNOS was a step forward in benefiting the candidates on the waiting list, there are a number of other factors that have significant impact on waiting times.

Our results note that those with lower serum sodium did have a shorter waiting time compared to candidates with higher sodium. Portal hypertension due to cirrhosis is widely believed to induce systemic and splanchnic vasodilation, with subsequent activation of the renin-angiotensin-aldosterone system and 
Table 2 Variables included in the final model

\begin{tabular}{|c|c|c|c|c|c|}
\hline Variable & $t$-score & P-value & Variable & $t$-score & $\mathrm{P}$-value \\
\hline Patient demographics & & & Blood group & & \\
\hline Age & -5.86 & $<0.001$ & A & 22.47 & $<0.001$ \\
\hline Female sex & 3.15 & 0.002 & $\mathrm{AB}$ & 16.07 & $<0.001$ \\
\hline BMI & 3.48 & $<0.0 \mathrm{~s} 01$ & $\mathrm{O}$ & 22.5 & $<0.001$ \\
\hline Ethnicity & & & Liver/kidney transplant & 24.14 & $<0.001$ \\
\hline Hispanic & 3.13 & 0.002 & Listing region & & \\
\hline Caucasian & 2.31 & 0.021 & 1 & 21.92 & $<0.001$ \\
\hline African American & 1.84 & 0.066 & 2 & 19.73 & $<0.001$ \\
\hline Biochemical & & & 3 & -8.57 & $<0.001$ \\
\hline Bilirubin & -91.08 & $<0.001$ & 4 & 17.76 & $<0.001$ \\
\hline INR & -62.08 & $<0.001$ & 6 & 11.72 & $<0.001$ \\
\hline Albumin & 6.65 & $<0.001$ & 7 & 18.40 & $<0.001$ \\
\hline Sodium & 15.96 & $<0.001$ & 8 & 14.37 & $<0.001$ \\
\hline Other disease factors & & & 9 & 18.57 & $<0.001$ \\
\hline Ascites & -6.55 & $<0.001$ & Source of pay & & \\
\hline TIPS & 6.3 & $<0.001$ & Private & 2.52 & 0.012 \\
\hline Ventilator support & -44.9 & $<0.001$ & Medicaid & 2.39 & 0.017 \\
\hline Other life support & -7.01 & $<0.001$ & MedicareFFS & 3.32 & 0.001 \\
\hline \multirow[t]{3}{*}{ HCC } & -5.66 & $<0.001$ & MedicareCh & 2.04 & 0.041 \\
\hline & & & Department/VA & 4.01 & $<0.001$ \\
\hline & & & Other & 2.82 & 0.005 \\
\hline
\end{tabular}

BMI, body mass index; INR, international normalized ratio; TIPS, transjugular intrahepatic portosystemic shunt; HCC, Hepatocellular carcinoma; VA, Veterans Affairs

hyponatremia [10]. In similar respects, hyponatremia has been found to be an early detector of kidney dysfunction in cirrhotic patients, and therefore increases mortality on the waiting list $[11,12]$. The severity of hyponatremia may be associated with the overall disease severity of cirrhosis and end-stage liver disease, as well as declining renal function, impacting the MELD score. Studies have now shown that adding sodium to the MELD model may have improved prognostic values $[11,13]$. Likewise, hypoalbuminemia is associated with a shorter waiting list time. Since albumin is an indication of nutritional status, at increasingly lower levels it may be a result of declining hepatic synthetic function, which would correlate with elevated INR. Although Merli et al did not find albumin to be an independent risk factor for mortality among cirrhotic patients, other studies have found that for every $1 \mathrm{~g}$ reduction in serum albumin, the risk of death increases by $45 \%$, making it a key predictor of mortality on the liver transplantation waiting list, especially among those with MELD $<15[8,13,14]$. This finding warrants consideration if including serum albumin levels in future models for liver allocation will help capture patients who have severe disease, but is not reflected in serum bilirubin, creatinine, and INR.

Other disease severity factors also contribute to the waiting time. MELD exception points and current mechanical ventilation accounted for the disease severity and reflect the need for emergent liver transplantation. Complications of cirrhosis, such as the presence and degree of ascites, also decrease the waiting time. In addition, the presence of hepatopulmonary syndrome, portopulmonary hypertensions, variceal bleeding, and infection, which can lead to poorer prognosis, need to be further elucidated and accounted for in the allocation of livers [15]. MELD exception points for HCC, and giving higher priority to patients on mechanical ventilation/other life support, represent attempts to decrease waiting-list mortality with the severity-driven model. A recent validation of MELD $>40$ showed that these candidates actually have higher waiting-list mortality than those with HCC, despite the higher overall score among patients with HCC due to the exception points [16]. This indicates a need to reassess significant factors that can identify more candidates who would benefit from early transplantation, thus improving survival on the waitlist.

An interesting finding of our analysis was that patients who had undergone a TIPS procedure waited longer for liver transplantation, especially considering that these patients are acutely bleeding or have refractory ascites. Patients who are actively bleeding, hemodynamically stable, and undergo an urgent TIPS procedure have a higher risk of complications [17]. Because these candidates become "too sick", they may drop off the transplant list and never receive an organ. Additionally, 
elective TIPS is generally reserved for patients with MELD $<18$, as this is associated with better outcomes and improved survival compared to those with MELD >18 [18]. Another reason for increased waiting time for patients with TIPS placement could perhaps be related to an improvement of ascites and in turn renal function post TIPS placement, which would drive the MELD score lower than before.

Several factors other than disease severity also contributed to waiting time. Older patients received liver transplantation sooner than their younger counterparts. This may be due to multiple factors, including liver diseases that manifest in middle-aged adults as opposed to young adults, increased likelihood of decompensation in older patients who have had liver disease for longer, and sizing of livers for transplantation. African Americans were also noted to have shorter waiting times on the liver transplantation list. Although the reasons are unclear and likely multifactorial, one partial explanation may be the increased baseline creatinine typically seen in African Americans because of their higher muscle mass.

Transplant region is an important predictor: each region had significantly different transplant times, which might be a result of increased demands in certain locations or may

\section{Summary Box}

\section{What is already known:}

- Demand for liver transplantation surpasses the donors available in the United States, with approximately $50 \%$ of candidates listed receiving an organ in 2016

- Scores, such as the model for end-stage liver disease (MELD) and Child-Turcotte-Pugh, have been widely used to predict mortality in liver disease and severity

- In 2002, UNOS adopted MELD to determine the allocation of liver transplants

\section{What the new findings are:}

- Markers of liver disease severity, such as the components of MELD, sodium, albumin, and complications of decompensated liver disease (e.g. ascites, need for mechanical ventilation) predicted shorter waiting times for a liver transplant

- Patients who underwent transjugular intrahepatic portosystemic shunt placement waited longer on the transplant list, possibly because of their improved overall disease severity

- Candidates living in region 3 were likely to receive liver transplants sooner because the donor organs available exceeded the number of candidates listed

- ABO blood type and primary payer status also affect time spent on the liver transplant list reflect the distribution of certain large liver transplant centers. Studies have found that candidates from rural areas were registered less and received fewer transplants, most likely because of either their distance from a transplant center or their decreased likelihood of completing a pre-transplantation assessment $[19,20]$. In particular, we found that those living in region 3 had the shortest time on the waiting list. This was actually because the number of livers available for transplant in 2016 exceeded the number of registrations in that region. Another important factor was $\mathrm{ABO}$ blood group. It would be expected that patients with less common blood types would wait longer on the transplant list, although transplantation across $\mathrm{ABO}$ groups are performed in some emergent circumstances. Given the heterogeneity in the distribution of $\mathrm{ABO}$ groups in the population, the availability of organs will be different, resulting in varied waiting times. Another interesting finding in our study was the variation in waiting times depending on primary payer status. Little is known about how primary payer status can affect waiting times for liver transplantation and further studies are needed in this regard.

The severity-driven model that UNOS adopted almost two decades ago made great strides in decreasing mortality on the waiting list. However, as this study and previous reports have indicated, other factors still need to be considered that impact both waiting time and the survival of liver transplant candidates $[21,22]$. In summary, MELD is an important part of predicting the waiting time for transplantation; however, it is not the only determinant. Further research is needed to address the reasons for the variation in waiting times among demographics, regions, $\mathrm{ABO}$ blood group types, and payment systems in an attempt to decrease waiting times, standardize the listing process, and improve patient outcomes.

\section{References}

1. Freeman RB, Wiesner RH, Edwards E, Harper A, Merion R, Wolfe R; United Network for Organ Sharing Organ Procurement and Transplantation Network Liver and Transplantation Committee. Results of the first year of the new liver allocation plan. Liver Transpl 2004;10:7-15.

2. Brown RS Jr, Lake JR. The survival impact of liver transplantation in the MELD era, and the future for organ allocation and distribution. Am J Transplant 2005;5:203-204.

3. Transplant trends. Available from: https://www.unos.org/data/ transplant-trends [Accessed 6 November 2017]

4. Malinchoc M, Kamath PS, Gordon FD, Peine CJ, Rank J, ter Borg PC. A model to predict poor survival in patients undergoing transjugular intrahepatic portosystemic shunts. Hepatology 2000;31:864-871.

5. Kamath PS, Wiesner RH, Malinchoc M, et al. A model to predict survival in patients with end-stage liver disease. Hepatology 2001;33:464-470.

6. Borroni G, Maggi A, Sangiovanni A, Cazzaniga M, Salerno F. Clinical relevance of hyponatraemia for the hospital outcome of cirrhotic patients. Dig Liver Dis 2000;32:605-610.

7. Porcel A, Díaz F, Rendón P, Macías M, Martín-Herrera L, GirónGonzález JA. Dilutional hyponatremia in patients with cirrhosis and ascites. Arch Intern Med 2002;162:323-328. 
8. Merli M, Riggio O, Dally L. Does malnutrition affect survival in cirrhosis? PINC (Policentrica Italiana Nutrizione Cirrosi). Hepatology 1996;23:1041-1046.

9. Alberino F, Gatta A, Amodio P, et al. Nutrition and survival in patients with liver cirrhosis. Nutrition 2001;17:445-450.

10. John S, Thuluvath PJ. Hyponatremia in cirrhosis: pathophysiology and management. World J Gastroenterol 2015;21:3197-3205.

11. Ruf AE, Kremers WK, Chavez LL, Descalzi VI, Podesta LG, Villamil FG. Addition of serum sodium into the MELD score predicts waiting list mortality better than MELD alone. Liver Transpl 2005;11:336-343.

12. Kim WR, Biggins SW, Kremers WK, et al. Hyponatremia and mortality among patients on the liver-transplant waiting list. N Engl J Med 2008;359:1018-1026.

13. Myers RP, Tandon P, Ney M, et al. Validation of the five-variable Model for End-stage Liver Disease (5vMELD) for prediction of mortality on the liver transplant waiting list. Liver Int 2014;34:1176-1183.

14. Gunsar F, Raimondo ML, Jones S, et al. Nutritional status and prognosis in cirrhotic patients. Aliment Pharmacol Ther 2006;24:563-572.

15. Bernardi M, Gitto S, Biselli M. The MELD score in patients awaiting liver transplant: strengths and weaknesses. I Hepatol 2011;54:1297-1306.
16. Sharma P, Schaubel DE, Gong Q, Guidinger M, Merion RM. Endstage liver disease candidates at the highest model for end-stage liver disease scores have higher wait-list mortality than status-1A candidates. Hepatology 2012;55:192-198.

17. Bañares R, Casado M, Rodríguez-Láiz JM, et al. Urgent transjugular intrahepatic portosystemic shunt for control of acute variceal bleeding. Am J Gastroenterol 1998;93:75-79.

18. Ferral H, Gamboa P, Postoak DW, et al. Survival after elective transjugular intrahepatic portosystemic shunt creation: prediction with model for end-stage liver disease score. Radiology 2004;231:231-236.

19. Axelrod DA, Guidinger MK, Finlayson S, et al. Rates of solid-organ wait-listing, transplantation, and survival among residents of rural and urban areas. JAMA 2008;299:202-207.

20. Goldberg DS, French B, Forde KA, et al. Association of distance from a transplant center with access to waitlist placement, receipt of liver transplantation, and survival among US veterans. JAMA 2014;311:1234-1243.

21. Cholongitas E, Burroughs AK. The evolution in the prioritization for liver transplantation. Ann Gastroenterol 2012;25:6-13.

22. Merion RM, Schaubel DE, Dykstra DM, Freeman RB, Port FK, Wolfe RA. The survival benefit of liver transplantation. Am J Transplant 2005;5:307-313. 